\title{
Monte Carlo radiative transfer in SPH density fields ${ }^{\star}$
}

\author{
D. Stamatellos and A. P. Whitworth
}

\author{
School of Physics \& Astronomy, Cardiff University, 5 The Parade, Cardiff CF24 3YB, Wales, UK \\ e-mail: D.Stamatellos@astro.cf.ac.uk
}

Received 28 February 2005 / Accepted 25 April 2005

\begin{abstract}
We combine a Monte Carlo radiative transfer code with an SPH code, so that - assuming thermal equilibrium - we can calculate dust-temperature fields, spectral energy distributions, and isophotal maps, for the individual time-frames generated by an SPH simulation. On large scales, the radiative transfer cells (RT cells) are borrowed from the tree structure built by the SPH code, and are chosen so that their size - and hence the resolution of the calculated temperature field - is comparable with the resolution of the density field. We refer collectively to these cubic RT cells as the global grid. The code is tested and found to treat externally illuminated dust configurations very well. However, when there are embedded discrete sources, i.e. stars, these produce very steep local temperature gradients which can only be modelled properly if - in the immediate vicinity of, and centred on, each embedded star - we supplement the global grid with a star grid of closely spaced concentric RT cells.
\end{abstract}

Key words. ISM: clouds - ISM: structure - ISM: dust, extinction - methods: numerical - radiative transfer - hydrodynamics

\section{Introduction}

Smoothed Particle Hydrodynamics (SPH) (Lucy 1977; Gingold \& Monaghan 1977) is a Lagrangian computational method which invokes a large array of particles to describe a fluid, assigns properties such as mass, position and velocity to each particle, and estimates intensive thermodynamic variables like density and pressure (and their derivatives) using local averages (for reviews see Benz 1990, 1991; Monaghan 1992). The main advantage of this method is that, in principle, there are no limitations on the geometry of the system or how far it can evolve from the initial conditions.

Because of the large computational cost incurred when radiative transfer is modelled fully in three dimensions, many previous treatments have been limited to one or two dimensions (e.g. Efstathiou \& Rowan-Robinson 1990, 1991; Men'shchikov \& Henning 1997; Zucconi et al. 2001), and radiative transfer has only recently been included in SPH simulations, where a fully three-dimensional treatment is essential (Kessel-Deynet \& Burkert 2000; Oxley \& Woolfson 2003; Whitehouse \& Bate 2004; Viau et al. 2005). The Monte Carlo approach for equilibrium radiative transfer (Lefevre et al. 1982, 1983; Wolf et al. 1999) is well suited to systems with arbitrary geometries, but, as with any Monte Carlo method, it is computationally expensive, especially when iteration is required. Recently, Bjorkman \& Wood (2001), extended an idea by Lucy (1999) and proposed a method to avoid iteration, by remitting photons as soon as they are absorbed, with a frequency distribution adjustment technique. This method avoids iteration, and it is fast when

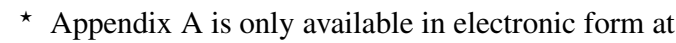
http://www. edpsciences.org compared with the traditional Monte Carlo radiative transfer methods. It has been applied successfully to a variety of problems, such as protoplanetary discs (e.g. Wood et al. 2002), prestellar cores (e.g. Stamatellos et al. 2004) and Class I objects (e.g. Whitney et al. 2003).

In this paper, we develop and test a method which uses Monte Carlo radiative transfer with frequency distribution adjustment, to calculate the dust-temperature field, the spectral energy distribution (SED), and isophotal maps, for individual time-frames from SPH simulations. We use the SPH tree to construct a grid of cubic radiative transfer cells (hereafter RT cells) with which the photons interact; we refer to this grid as the global grid. The global grid obtained in this way is similar to the tree-structured adaptive grid invoked by Kurosawa \& Hillier (2001), but because it borrows the tree structure already derived as part of the SPH code, it minimizes the computational burden that will be added to the hydrodynamic simulations when the two methods are combined. A similar method has been used by Oxley \& Woolfson (2003), but they adopt an average, wavelength-independent opacity. To account for the large temperature gradients that are expected very close to stars, we supplement the global grid described above with a grid of concentric spherical radiative transfer cells around each star; we refer to this supplementary grid as the star grid.

\section{Monte Carlo radiative transfer with frequency distribution adjustment}

The Monte Carlo radiative transfer method (e.g. Stamatellos \& Whitworth 2003) uses a large number of monochromatic luminosity packets ( $L$-packets) to represent the radiation injected 
into the medium (e.g. stellar radiation, cooling radiation, background radiation). Each $L$-packet is given a random frequency, $v$, and a random optical depth, $\tau$, which determines its free path, i.e. how far it travels before interacting with the medium. The medium itself is divided into a number of RT cells, each with uniform density and temperature. Each time an $L$-packet is scattered, it is given a new random direction, using the Henyey \& Greenstein (1941) scattering phase function, and a new random optical depth, $\tau$. Each time an $L$-packet is absorbed, it raises the temperature of the RT cell in which it is absorbed from $T$ to $T+\Delta T$, and the packet is immediately reemitted isotropically, so that the dust is in radiative equilibrium. The temperature of the RT cell is computed by equating the total absorbed luminosity to the total emitted luminosity. The frequency of the reemitted $L$-packet is chosen using a probability distribution function (PDF) constructed from the difference between the emissivity of the cell before and after absorption of the $L$-packet (Bjorkman \& Wood 2001; Baes et al. 2005),

$$
\begin{aligned}
p(v) \mathrm{d} v & =\frac{\kappa_{v}\left[B_{v}(T+\Delta T)-B_{v}(T)\right] \mathrm{d} v}{\int_{0}^{\infty} \kappa_{v}\left[B_{v}(T+\Delta T)-B_{v}(T)\right] \mathrm{d} v} \\
& \simeq \frac{\kappa_{v} B^{\prime}(T) \mathrm{d} v}{\int_{0}^{\infty} \kappa_{v} B^{\prime}(T) \mathrm{d} v},
\end{aligned}
$$

where $B^{\prime}(T) \equiv \mathrm{d} B_{v} / \mathrm{d} T$. At the same time the $L$-packet is given a new random optical depth, $\tau$. Using the PDF of Eq. (1), the correct temperature distribution and spectrum of the system are obtained without iteration, once all the $L$-packets have been propagated through the medium and escaped. The method accounts for both absorption and scattering, it is robust, it is very accurate (see tests by Bjorkman \& Wood 2001; Stamatellos \& Whitworth 2003), it can readily be parallized, and it can treat systems with arbitrary geometries.

\subsection{Construction of radiative transfer cells}

The radiative transfer cells must be constructed so that their linear size is less than, or on the order of, the local directional temperature- and density- scale-heights (e.g. $\Delta x \lesssim \operatorname{MIN}\left\{|\mathrm{d} \ln [\rho] / \mathrm{d} x|^{-1},|\mathrm{~d} \ln [T] / \mathrm{d} x|^{-1}\right\}$, see Stamatellos \& Whitworth 2003). This ensures that the temperature and density do not vary too greatly between adjacent RT cells. The construction of the RT cells depends on the specific system under study, and, in an hydrodynamic simulation, where the system changes from step to step, the RT cells need to be reconstructed at every step. Therefore, a robust and efficient algorithm for the construction of RT cells is required.

To construct RT cells we take advantage of the fact that our SPH code calculates gravitational forces with the aid of a spatial tessellation tree (Barnes \& Hut 1986; Hernquist \& Katz 1989). The whole computational domain is contained within a cubic cell, the root cell, which is then divided into eight smaller cubic cells of equal size. If any of these cells contains more than one particle, it is subdivided into eight even smaller cells. This procedure continues recursively until the smallest cells each contain just one particle or no particles at all. The resulting ensemble of cells constitutes the SPH tree. In SPH, the tree is used so that distant particles do not contribute individually to the local gravitational field, but collectively through the cell in which they are contained; the tree is also used to find neighbouring particles so that smoothed values of thermodynamic variables and their derivatives can be evaluated.

The global grid then comprises the smallest set of RT cells which (a) spans the entire computational domain (or at least that part of it containing matter) and (b) contains fewer than $N_{\text {MAX }}$ SPH particles. If $N_{\text {MAX }}$ is chosen to be of the same order as $N_{\text {NEIB }}$ (the number of neighbour particles used for smoothing purposes), then the RT cells are necessarily similar in size to the resolution of the SPH simulation. Specifically we choose $N_{\mathrm{MAX}} / N_{\mathrm{NEIB}} \sim 2$ to 3 , so that each RT cell has linear size $\sim 2 h$ (where $h$ is the SPH smoothing length) and contains $\sim 0.3 N_{\text {NEIB }}$ particles.

This method of constructing RT cells is similar to the method of Kurosawa \& Hiller (2001) (see also Kurosawa et al. 2004), but it is implemented using the Barnes \& Hut (1986) tree structure. Both methods produce RT cells with approximately equal mass. It is also similar to the method used by Oxley \& Woolfson (2003), but they construct RT cells with size $1 / 3$ to $2 / 3$ of the SPH smoothing length. Our method has the advantage that we can readily compute the density in an RT cell from the number of particles in the cell and the cell size, whereas in the Oxley \& Woolfson method the density must be computed by an SPH sum over the neighbouring particles, and this is computationally expensive.

The method we use to construct RT cells, is implemented relatively easily within SPH and exploits information about the tree already calculated for SPH purposes. Thus, it does not add much to the code running time. It also furnishes us with an efficient procedure for finding which RT cell an $L$-packet is in. This is critical for the efficiency of the code, because as an $L$-packet propagates through the computational domain, this search procedure is performed many times.

\subsection{L-packet propagation and interaction}

The propagation of $L$-packets through the computational domain is performed in small steps, $\delta S$, which gradually decrease the optical depth $\tau$ of the $L$-packet until $\tau=0$, whereupon the $L$-packet reaches an interaction point. This is the most computationally expensive part of the code, particularly as there is no analytical expression for the density - and hence the opacity - along the path of an $L$-packet. Instead, the density is determined by identifying the RT cell through which the $L$-packet is passing. After each step $\delta S$, we use the SPH tree to find which RT cell $i$ the $L$-packet is now in. The search starts from the root cell and proceeds to lower level cells, until the required RT cell is reached. The search contributes a significant fraction of the code running time, and therefore, it is essential to choose the step $\delta S$ appropriately. We use

$\delta S=\operatorname{MIN}\left\{\eta_{1} S_{i}, \eta_{2} \bar{\ell}_{i}, \tau \bar{\ell}_{i}, \eta_{3}|\boldsymbol{r}|\right\}$,

where $\eta_{1}, \eta_{2}, \eta_{3}$ are constants in the range $(0.1,1)$ and determine the accuracy; $S_{i}$ is the linear extent of RT cell $i ; \bar{\ell}_{i}=\kappa_{\lambda} \rho_{i}$ is the mean-free-path for the $L$-packet ( $\kappa_{\lambda}$ is the monchromatic mass-opacity at the wavelength of the $L$-packet, and $\rho_{i}$ is the 
density in cell $i) ; \boldsymbol{r}$ is the position of the photon relative to any discrete source (i.e. star).

Kurosawa \& Hiller (2001) adopt a more detailed approach, in which they use the path of an $L$-packet to determine where it intercepts the boundaries of the RT cells through which it passes. Our method is simpler to implement, it propagates the $L$-packets efficiently (usually the propagation step is on the order of the local RT cell size), and it is accurate (due to the fact that neighbouring RT cells have similar densities).

\section{Tests}

We consider a spherical cloud with uniform density $n=8.6 \times$ $10^{4} \mathrm{~cm}^{-3}$, mass $M=100 M_{\odot}$ and radius $R=3.5 \times 10^{4} \mathrm{AU}$, and represent it with randomly distributed SPH particles. We then test the method of constructing the global grid and propagating $L$-packets through the grid in three ways. (i) We use the thermodynamic equilibrium test, where the cloud is bathed in an isotropic blackbody radiation field with a given temperature (Stamatellos \& Whitworth 2003). (ii) We illuminate the cloud with the Black (1994) interstellar radiation field (BISRF) and compare our results with the results obtained previously using a spherically symmetric grid of concentric cells (Stamatellos \& Whitworth 2003). (iii) We embed a low-temperature star at the centre of the cloud and again compare the results with those obtained previously using a spherically symmetric grid of concentric cells (see online appendix). We present the results in the following subsections.

\subsection{Cloud bathed in a blackbody radiation field}

We use $N_{\text {тотAL }}=20000 \mathrm{SPH}$ particles and set the maximum number of particles in an RT cell to $N_{\text {MAX }}=60$, giving $N_{\text {CELLS }}=$ 1484 RT cells, each containing on average $\bar{N} \sim 15$ SPH particles. The density in each RT cell is plotted as a function of radius in Fig. 1a. Because the SPH particles are distributed randomly, there are statistical variations in the density at the $\pm \bar{N}^{-1 / 2} \simeq \pm 25 \%$ level. There are also a number of RT cells that appear to be outside the boundary of the cloud and to have very low density. In reality, only a small part of each of these RT cells is within the cloud and therefore they contain only a very small number of particles. These irregular RT cells are a side effect of representing a spherical cloud with a grid of cubic cells. The same problem was also encountered by Oxley \& Woolfson (2001).

$10^{8} \mathrm{~L}$-packets are then injected from random positions on the boundary of the cloud, and with random frequencies and injection angles, so as to imitate an isotropic blackbody radiation field at $15 \mathrm{~K}$ (see Stamatellos et al. 2004). The cloud should then adopt the temperature of the illuminating radiation field, i.e. $15 \mathrm{~K}$. The computed temperature of each RT cell is plotted on Fig. 1b. In the body of the cloud the temperature is very close to $15 \mathrm{~K}$, with the error being less than $\pm 3 \%$. The errors are higher near and outside the boundary of the cloud, because of the presence of the irregular RT cells. These errors can be reduced by increasing the number of SPH particles, and/or the number of RT cells, and/or the number of $L$-packets.
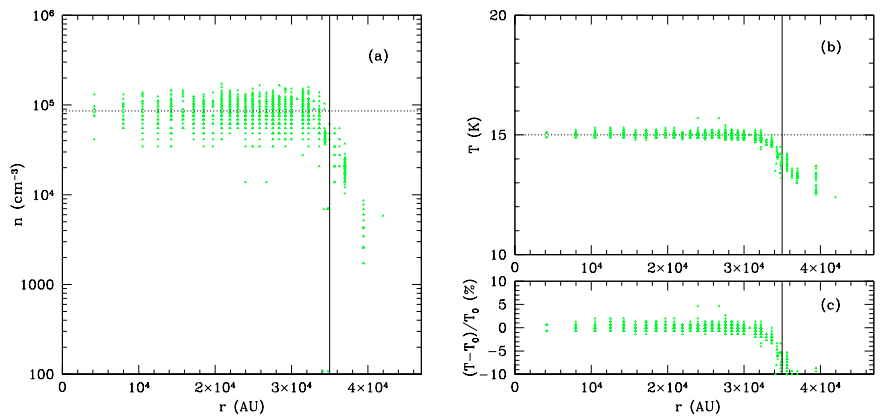

Fig. 1. A uniform-density spherical cloud, represented by 20000 SPH particles, is illuminated by an isotropic blackbody radiation field at $T=15 \mathrm{~K}$, using $10^{8} \mathrm{~L}$-packets. RT cells are constructed with $N_{\text {MAX }}=60$, giving $N_{\text {CELLS }}=1484$. a) Triangles give the densities of the individual RT cells, the horizontal dotted line marks the mean density, and the vertical line marks the boundary of the cloud. b) Triangles give the temperatures of the individual RT cells, the horizontal dotted line marks $T=15 \mathrm{~K}$, and the vertical line marks the boundary of the cloud. c) The percentage error in the computed temperature.
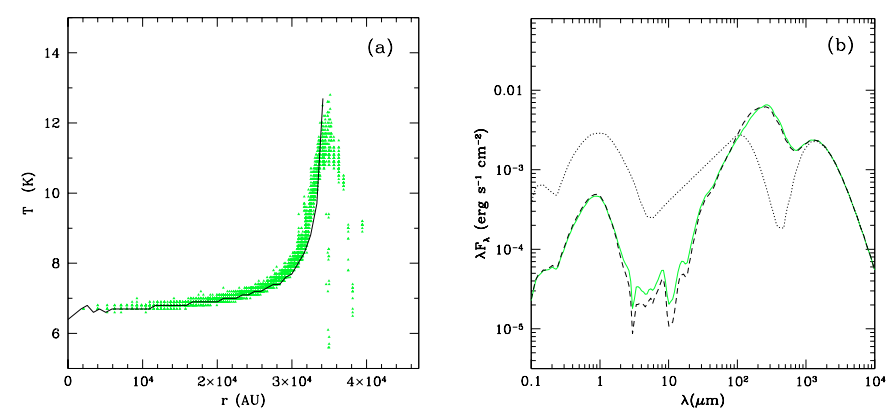

Fig. 2. A uniform-density spherical cloud, represented by 200000 SPH particles, is illuminated by the BISRF radiation field, using $10^{8} L$-packets. RT cells are constructed with $N_{\mathrm{MAX}}=100$ giving $N_{\text {CELLS }}=11862$. a) Triangles give the temperatures of the individual RT cells and the solid line gives the accurate temperature profile. b) The computed SED (solid line) is compared with the accurate SED (dashed line) and the illuminating BISRF (dotted line).

\subsection{Cloud embedded in the ISRF}

We use $N_{\text {TOTAL }}=200000 \mathrm{SPH}$ particles and set the maximum number of particles in an RT cell to $N_{\text {MAX }}=100$, giving $N_{\text {CELLS }}=$ 11862 RT cells, each containing on average $\bar{N} \sim 18$ SPH particles. $10^{8} L$-packets are then injected from random positions on the boundary of the cloud, and with random frequencies and injection angles, so as to imitate the isotropic Black (1994) interstellar radiation field (BISRF). The advantage of this test is that the BISRF covers a wide wavelength range, including regions where scattering dominates (UV and optical) and regions where absorption/emission dominates (IR and submm). Therefore, we can test the validity of the method over a wide range of wavelengths. Figure 2 compares the resulting temperature profile and SED with accurate results obtained using a spherically symmetric grid of thin concentric RT cells. The agreement is very good, apart from the irregular RT cells at the boundary. 


\section{Stars in SPH-RT simulations}

The presence of a normal- or high-temperature star in an SPH simulation makes the treatment of radiation transport more complicated, firstly because in the immediate vicinity of the star the dust is destroyed by sublimation and/or chemical sputtering (e.g. Lenzuni et al. 1995), and secondly because just outside this region there are very large temperature gradients which cannot be captured by the global grid.

\subsection{Dust destruction radius}

The dust destruction temperature is estimated to be between $T_{\text {DEST }} \sim 800 \mathrm{~K}$ and $T_{\text {DEST }} \sim 2100 \mathrm{~K}$ (Lenzuni et al. 1995; Duschl et al. 1996) and depends on the assumed dust composition. The heating rate for an isolated dust grain at distance $R$ from a star having radius $R_{\star}$ and surface temperature $T_{\star}$ is

$\mathcal{G}=\pi r_{\mathrm{d}}^{2} \int_{0}^{\infty} Q_{v}\left(\frac{R_{\star}}{R}\right)^{2} \pi B_{v}\left(T_{\star}\right) \mathrm{d} \nu$,

where $r_{\mathrm{d}}$ is the radius of the grain, and $Q_{v}$ is the absorption efficiency. Similarly the dust cooling rate is

$\mathcal{L}=4 \pi r_{\mathrm{d}}^{2} \int_{0}^{\infty} Q_{v} \pi B_{v}(T) \mathrm{d} v$

where $T$ is the dust temperature. Assuming that the dust is in thermal equilibrium, i.e. $\mathcal{G}=\mathcal{L}$, and putting $Q_{v} \propto v^{\beta}(1 \lesssim \beta \lesssim$ 2), we obtain

$T=\left(\frac{R_{\star}}{2 R}\right)^{2 /(4+\beta)} T_{\star}$

At the dust destruction radius, $T=T_{\mathrm{DEST}}$, and hence $R=R_{\mathrm{DEST}}$, where

$R_{\mathrm{DEST}}=\frac{R_{\star}}{2}\left(\frac{T_{\star}}{T_{\mathrm{DEST}}}\right)^{(4+\beta) / 2}$.

In the optically thin limit, this radius defines the spherical volume around the star which should be devoid of dust. If the envelope of dust around the star is optically thick to its own cooling radiation, it will act to trap the radiation from the star and $R_{\mathrm{DEST}}$ will be somewhat larger. The values of $R_{\mathrm{DEST}}$ stipulated by Ivezić et al. (1997; see below) take this into account.

\subsection{Steep temperature gradients in the vicinity of a star}

The density and temperature gradients close to a star are large, and can only be resolved with RT cells whose radial extent is comparable with, or smaller than, the dust destruction radius $R_{\mathrm{DEST}}$. Unless an impractically large number of SPH particles has been used in the simulation, the cubic RT cells of the global grid (with size $\sim h$, where $h$ is the smoothing length of the SPH kernel) are much larger than $R_{\mathrm{DEST}}$, as illustrated schematically in Fig. 3. Thus the steep temperature gradients in the vicinity of the star occupy only a few RT cells of the global grid, and they are not properly resolved. Consequently the temperatures very close to the star are underestimated.

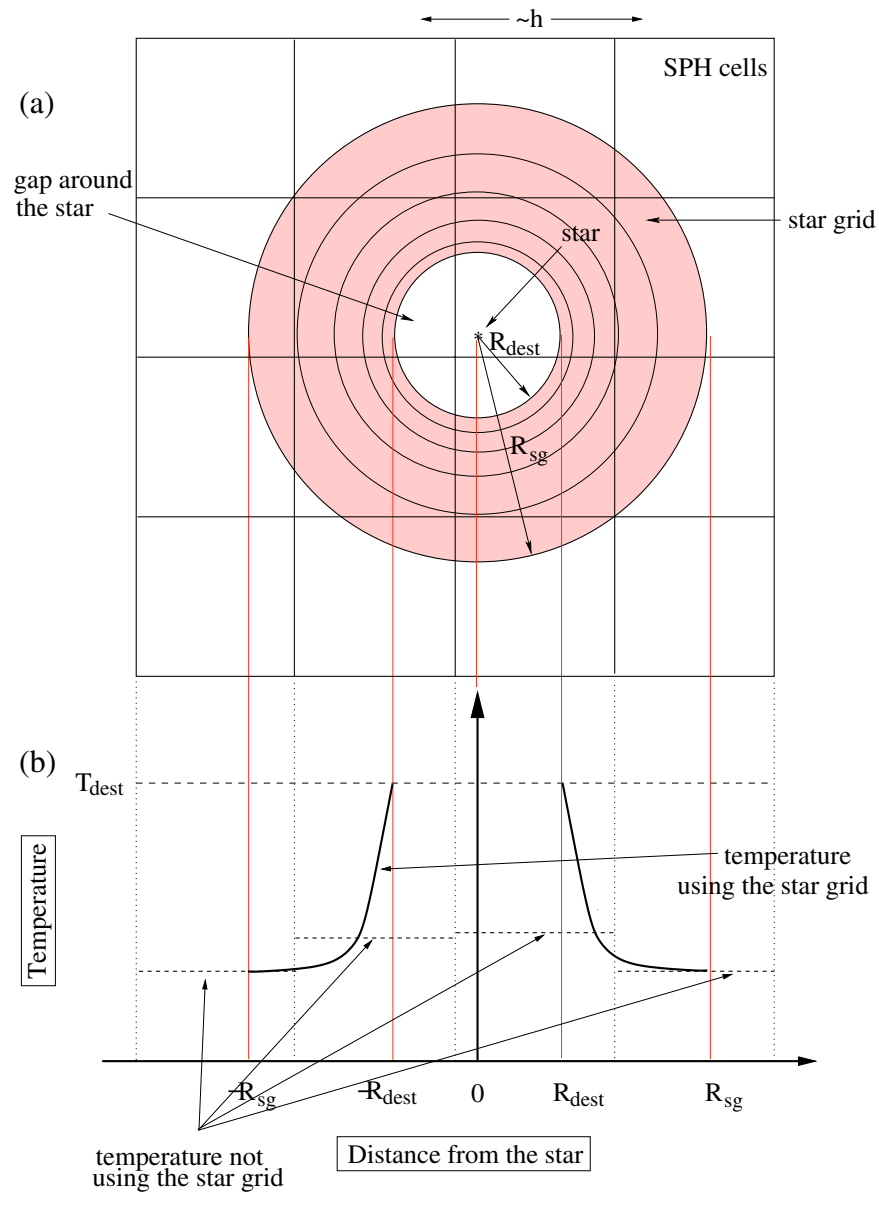

Fig. 3. a) The square cells represent the global grid derived from the SPH tree, and the concentric circles represent the additional star grid. b) The dashed horizontal lines show the temperature computed (inaccurately) using the global grid on its own, whilst the solid curve shows the (accurate) temperature profile computed using the additional star grid.

(Further out the temperature may be overestimated or underestimated, but this is less critical.) The upshot is that $L$-packets which are absorbed very close to the star are then re-emitted from a wavelength distribution corresponding to an inappropriately low temperature, and hence at inappropriately long wavelengths. This means that they are able to propagate further away from the star, or even to escape from the system altogether. Consequently the SED has a deficit of radiation at the short (mainly NIR) wavelengths emitted by hot dust, and a corresponding excess of radiation at long (mainly FIR) wavelengths. This problem also arises in the simulations of Kurosawa et al. (2004).

To resolve this problem, we construct an extra grid of concentric shells (a star grid) around each star (see Fig. 3). To construct a star grid, we must specify:

- The dust destruction radius, $R_{\mathrm{DEST}}$, which is determined by the temperature $T_{\star}$ and the radius $R_{\star}$ of the star. For simplicity we put

$$
L_{\star}=4 \pi R_{\star}^{2} \sigma T_{\star}^{4}=L_{\odot}\left(\frac{M_{\star}}{M_{\odot}}\right)^{3}+\frac{G M_{\star} \dot{M}_{\star}}{R_{\star}},
$$


where $M_{\star}$ is the mass of the star and $\dot{M}_{\star}$ is the accretion rate onto the star. The first term in Eq. (7) is the intrinsic luminosity of the star, and the second term is the accretion luminosity. For a Pre-Main Sequence star we can put $R_{\star} \simeq$ $3 R_{\odot}$ (Stahler 1988).

- The outer radius $R_{\mathrm{SG}}$, of the star grid, which should be on the order of the local SPH resolution, or, equivalently, on the order of the size of the local cubic RT cells of the global grid.

- The density profile between $R_{\mathrm{DEST}}$ and $R_{\mathrm{SG}}$, for which we adopt

$$
\rho=\rho_{0}\left(\frac{R_{\mathrm{DEST}}}{R}\right)^{p}, R_{\mathrm{DEST}}<R<R_{\mathrm{SG}},
$$

with $\rho_{0}$ fixed by conservation of mass, and $p=0$ to 2 .

Once all the above parameters have been specified, the star grid around each star is constructed by dividing up the space between $R_{\mathrm{DEST}}$ and $R_{\mathrm{SG}}$ into concentric RT cells of equal logarithmic width; typically $\sim 30$ RT cells are sufficient for each star grid. The complication that this introduces into an RT calculation is minimal. There is some overlap between the two sets of RT cells (the cubic cells of the global grid and the spherical cells of the star grid), but this does not create any major problems, as our tests in the next section demonstrate. $L$-packets which are inside the star grid interact with the RT cells of that grid, rather than the RT cells of the global grid.

However, we note that a spherically symmetric star grid may not be appropriate when the dust close to a star is concentrated in a disc. This situation can be handled with a simple extension to the method we have presented here, and it will be discussed in the companion paper (Stamatellos et al. 2005).

\section{Ivezić test}

We test the above method for including stars in radiative transfer calculations within SPH, using the configuration proposed by Ivezić et al. (1997), viz. a star with $T_{\star}=2500 \mathrm{~K}$ surrounded by a spherical envelope with an $R^{-2}$ density profile. We perform the test for envelopes having visual radial optical depths (from the centre to the edge of the envelope) $\tau_{\mathrm{V}}=1$ and $\tau_{\mathrm{V}}=100$ (see online appendix). For $\tau_{\mathrm{V}}=1$, we set $R_{\mathrm{DEST}}=9.11 R_{\star}$; and for $\tau_{\mathrm{V}}=100, R_{\mathrm{DEST}}=17.67 R_{\star}$, as stipulated by Ivezić et al. (1997). For both cases we set $R_{\mathrm{SG}}=20 R_{\mathrm{DEST}}$ and $R_{\text {OUTER }}=1000 R_{\text {DEST }}$ (where $R_{\text {OUTER }}$ is the outer radius of the envelope). Note that we do not need to specify $R_{\star}$.

We represent the envelope using 20000 SPH particles, distributed randomly so that they approximate to an $R^{-2}$ profile. (The approximation could be improved by settling the distribution to get rid of Poisson fluctuations, but we have not done this.) We use the SPH tree to construct a global grid with $N_{\text {MAX }}=55$ (which gives $N_{\text {CELLS }}=1276$ and $\bar{N}=15$ ). In addition, we have the option to introduce a star grid comprising 30 concentric RT cells, between $R_{\mathrm{DEST}}$ and $R_{\mathrm{SG}}$.

The results are compared with the very accurate results obtained using a one-dimensional spherically symmetric code in Stamatellos \& Whitworth (2003), hereafter "the benchmark calculation".
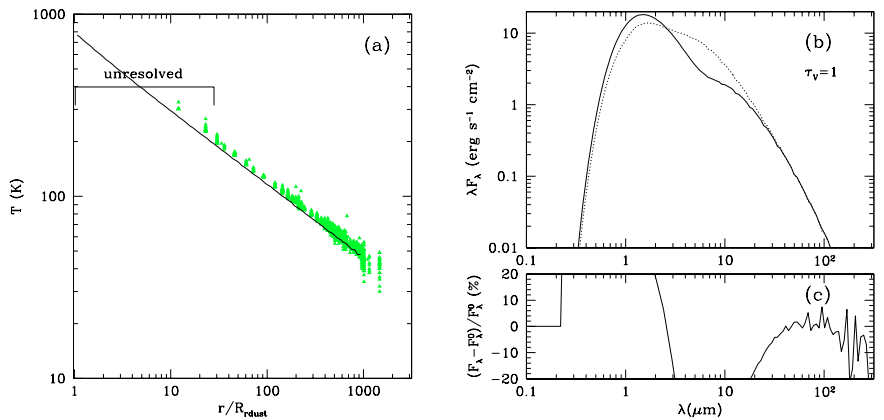

Fig. 4. Ivezić test with $\tau_{\mathrm{v}}=1$, using $5 \times 10^{7} L$-packets. The envelope is represented by $20000 \mathrm{SPH}$ particles and a global grid of $N_{\text {CELLS }}=1276$ RT cells (with $\bar{N}=15$ ), but no additional star grid around the star. a) The triangles represent the temperatures of the individual RT cells plotted against radius, whilst the solid line represents the benchmark calculation. The global grid on its own fails to capture the temperature profile close to the star. b) The solid line shows the SED computed here, and the dotted line shows the benchmark calculation. c) The percentage difference between the flux computed here and the benchmark calculation.
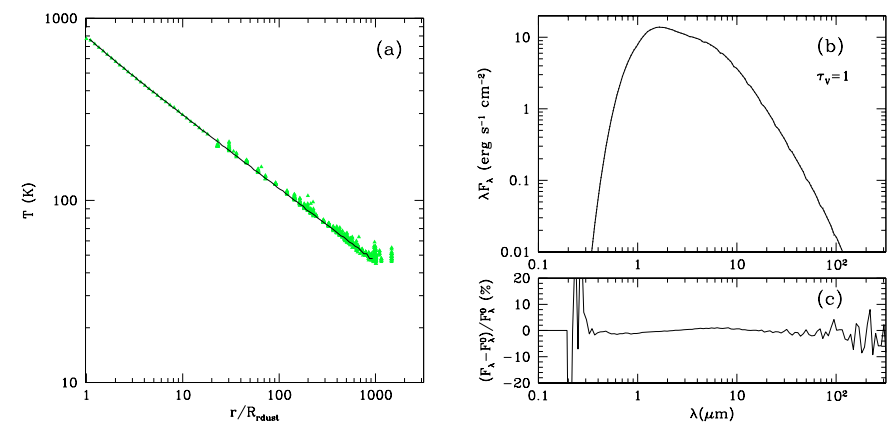

Fig. 5. Same as Fig. 4 but with an additional star grid around the star, i.e. a volume devoid of matter inside $R_{\mathrm{DEST}}$ and 30 concentric spherical RT cells between $R_{\mathrm{DEST}}$ and $R_{\mathrm{SG}}=20 R_{\mathrm{DEST}}$. Agreement with the benchmark calculation is now very good. Small differences are due to statistical noise.

We have performed the Ivezić test with $\tau_{\mathrm{V}}=1$ and $p=-2$, using $5 \times 10^{7} L$-packets, both without (Fig. 4), and with (Fig. 5), a star grid around the central star.

Without a star grid around the central star (Fig. 4) the cubic RT cells of the global grid in the vicinity of the star have size $\sim 20 R_{\mathrm{DEST}}$, and therefore they are unable to capture the large temperatures and densities there. This has two effects. First, the hottest dust is only at $\sim 300 \mathrm{~K}$ (rather than $\sim 800 \mathrm{~K}$ in the benchmark calculation), and consequently the SED shows a deficit of radiation at wavelengths around $8 \mu \mathrm{m}$. Second, the uniform density in the individual RT cells reduces the net radial optical depth, allowing more radiation to escape directly from the central star (specifically $\tau_{\mathrm{V}}^{\text {EFFECTVE }} \sim 0.3$ ); this shows up in the SED as an excess of radiation at wavelengths around $1 \mu \mathrm{m}$.

With a star grid around the central star (Fig. 5), the innermost RT cell (immediately outside $R_{\mathrm{DEST}}$ ) has radial extent $\sim 0.1 R_{\mathrm{DEST}}$, and the high temperatures and densities near the star are properly represented. Agreement with the benchmark calculation is then very good. Small differences are due to statistical noise in RT cells which do not intercept many $L$-packets. 


\section{Discussion}

In this paper we have presented a method for performing radiative transfer calculations on individual time-frames from SPH simulations. We use the SPH tree to construct a global grid of radiative transfer (RT) cells. This grid is constructed so that each RT cell contains a number of SPH particles close to the number of neighbours that each particle must have in SPH for smoothing purposes. The method thereby creates RT cells with size on the order of the local SPH smoothing length.

We test the method using the thermodynamic equilibrium test for a uniform-density spherical cloud, bathed in a blackbody radiation field. The method performs this test well. The global grid of RT cells that represent the cloud acquire the same temperature as the blackbody radiation field. The temperature is slightly underestimated near the boundary of the cloud due to the presence of RT cells that contain only a few SPH particles (irregular RT cells). The method also performs well for a uniform-density cloud illuminated by the interstellar radiation field.

We then examine a system comprising a low-temperature star embedded at the centre of a uniform-density spherical cloud. The temperature field and the SED are calculated with good accuracy. Very close to the star the temperature is slightly underestimated due to the fact that the temperature field there is not properly resolved. This in turn distorts the SED of the system slightly at short wavelengths. The problem is more pronounced when a normal- or high-temperature star is embedded in the cloud. The region very close to the star is then characterised by a very steep temperature gradient which cannot normally be captured by the cubic RT cells of the global grid, because these RT cells are too large.

To solve this problem we introduce an additional grid of concentric spherical RT cells around the star (the star grid). The star grid accounts for the dust-free region near the star, and its concentric spherical RT cells are small enough to capture steep temperature gradients, so that temperatures are calculated correctly close to the star. We test the use of a global grid in tandem with a star grid around the star, using the configuration proposed by Ivezić et al. (1997), and show that it performs very well, even if the optical depth is very large.

Our radiative transfer code can therefore be applied to arbitrary SPH density fields to produce dust temperature distributions, SEDs and isophotal maps at different wavelengths. These can then be used to compare the results of hydrodynamic simulations with observations. In the companion paper (Stamatellos et al. 2005) we apply this method to the collapse of turbulent molecular cores and the early stages of star formation.
Acknowledgements. We gratefully acknowledge support from the EC Research Training Network "The Formation and Evolution of Young Stellar Clusters" (HPRN-CT-2000-00155) and from PPARC (PPA/G/O/2002/00497). We would also like to thank J. Barnes for using his tree code (available online at http://www . ifa.hawaii. edu/ barnes/software.html).

\section{References}

Baes, M., Stamatellos, D., Davies, J., et al. 2005, New A, 10, 253

Barnes, J., \& Hut, P. 1986, Nature, 324, 446

Benz, W. 1990, Numerical Modelling of Nonlinear Stellar Pulsations Problems and Prospects, 269

Benz, W. 1991, Late Stages of Stellar Evolution. Computational Methods in Astrophysical Hydrodynamics, LNP Vol., 373, 259

Black, J. H. 1994, The First Symposium on the Infrared Cirrus and Diffuse Interstellar Clouds, ASP Conf. Ser., 58, 355

Bjorkman, J. E., \& Wood, K. 2001, ApJ, 554, 615

Duschl, W. J., Gail, H.-P., \& Tscharnuter, W. M. 1996, A\&A, 312, 624

Efstathiou, A., \& Rowan-Robinson, M. 1990, MNRAS, 245, 275

Efstathiou, A., \& Rowan-Robinson, M. 1991, MNRAS, 252, 528

Gingold, R. A., \& Monaghan, J. J. 1977, MNRAS, 181, 375

Henyey, L. C., \& Greenstein, J. L. 1941, ApJ, 93, 70

Hernquist, L., \& Katz, N. 1989, ApJS, 70, 419

Ivezić, Ž., Groenewegen, M. A. T., Men'shchikov, A., \& Szczerba, R. 1997, MNRAS, 291, 121

Kessel-Deynet, O., \& Burkert, A. 2000, MNRAS, 315, 713

Kurosawa, R., \& Hillier, D. J. 2001, A\&A, 379, 336

Kurosawa, R., Harries, T. J., Bate, M. R., \& Symington, N. H. 2004, MNRAS, 351, 1134

Lefevre, J., Bergeat, J., \& Daniel, J. Y. 1982, A\&A, 114, 341

Lefevre, J., Daniel, J. Y., \& Bergeat, J. 1983, A\&A, 121, 51

Lenzuni, P., Gail, H., \& Henning, T. 1995, ApJ, 447, 848

Lucy, L. B. 1977, AJ, 82, 1013

Lucy, L. B. 1999, A\&A, 344, 282

Men'shchikov, A. B., \& Henning, Th. 1997, A\&A, 318, 879

Monaghan, J. J. 1992, ARA\&A, 30, 543

Oxley, S., \& Woolfson, M. M. 2003, MNRAS, 343, 900

Stahler, S. W. 1988, ApJ, 332, 804

Stamatellos, D., \& Whitworth, A. P. 2003, A\&A, 407, 941

Stamatellos, D., Whitworth, A. P., André, P., \& Ward-Thompson, D. 2004, A\&A, 420, 1009

Stamatellos, D., Whitworth, A. P., Boyd, D. F. A., \& Goodwin, S. P. 2005, A\&A, 439, 159

Viau, S., Bastien, P., \& Cha, S.-H. 2004, in preparation

Whitehouse, S. C., \& Bate, M. R. 2004, MNRAS, 353, 1078

Whitney, B. A., Wood, K., Bjorkman, J. E., \& Wolff, M. J. 2003, ApJ, 591,1049

Wolf, S., Henning, T., \& Stecklum, B. 1999, A\&A, 349, 839

Wood, K., Lada, C. J., Bjorkman, J. E., et al. 2002, ApJ, 567, 1183

Zucconi, A., Walmsley, C. M., \& Galli, D. 2001, A\&A, 376, 650 
D. Stamatellos and A. P. Whitworth: Radiative transfer in SPH density fields, Online Material $p 1$

\section{Online Material}


D. Stamatellos and A. P. Whitworth: Radiative transfer in SPH density fields, Online Material p 2

\section{Appendix A: Supplementary tests}

\section{A.1. Low-temperature star surrounded by a spherical envelope}

We use $N_{\text {TOTAL }}=200000 \mathrm{SPH}$ particles and set the maximum number of particles in an RT cell to $N_{\text {MAX }}=60$, giving $N_{\text {CELLS }}=13050$ cells, each containing on average $\bar{N} \sim 16$ SPH particles. $10^{8} L$-packets are emitted by a central lowtemperature "star" having luminosity $L_{*}=7 L_{\odot}$ and surface temperature $T_{\star}=30 \mathrm{~K}$. Figure A. 1 compares the resulting temperature profile and SED with accurate results obtained using a spherically symmetric grid of thin concentric RT cells. The global grid used here has difficulty capturing the steep temperature gradient near the star. As a result the temperatures near the star are underestimated and the SED shows a deficit at short wavelengths. The temperature is also not very accurate near the boundary, due to the irregular RT cells there; and there are some small uncertainties in the SED at wavelengths longer than $2000 \mu \mathrm{m}$, due to the small number of $L$-packets generated at these wavelengths. Otherwise the agreement is good.

\section{A.2. Ivezić test with $\tau_{\mathrm{V}}=100$ and $p=-2$}

We have performed the Ivezić test with $\tau_{\mathrm{V}}=100$ and $p=$ -2 , using $10^{7} L$-packets, both without (Fig. A.2), and with (Fig. A.3), a star grid around the central star.

Without a star grid around the central star (Fig. A.2), the cubic RT cells of the global grid in the vicinity of the star are again unable to capture the large temperatures and densities there. Consequently the hottest dust is only at $\sim 200 \mathrm{~K}$ (rather than $\sim 800 \mathrm{~K}$ in the benchmark calculation). The SED is dominated by the emission from this dust, which peaks around $20 \mu \mathrm{m}$. In addition, the uniform densities in the RT cells reduces the effective radial optical depth $\left(\tau_{\mathrm{V}}^{\mathrm{EFECTIVE}} \sim 20\right)$ and so there is a small shoulder at $\sim 3 \mu \mathrm{m}$, due to radiation which escapes directly from the central star.

With a star grid around the central star (Fig. A.3) agreement with the benchmark calculation is very good. Small differences are again due to statistical noise. We emphasize that these calculations were performed with only $10^{7} \mathrm{~L}$-packets.
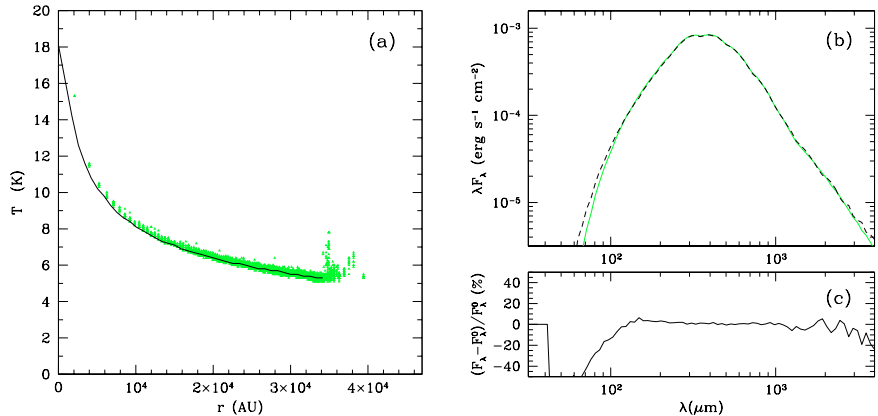

Fig. A.1. A uniform-density spherical cloud, represented by $200000 \mathrm{SPH}$ particles, is heated by a central low-temperature star with $L_{*}=7 L_{\odot}$ and $T_{*}=30 \mathrm{~K}$, which emits $10^{8} L$-packets. RT cells are constructed with $N_{\text {MAX }}=60$, giving $N_{\text {CELLS }}=13050$. a) Triangles give the temperatures of the individual RT cells and the solid line gives the accurate temperature profile. b) The computed SED (solid line) is compared with the accurate SED (dashed line). c) The precentage error in the SED.
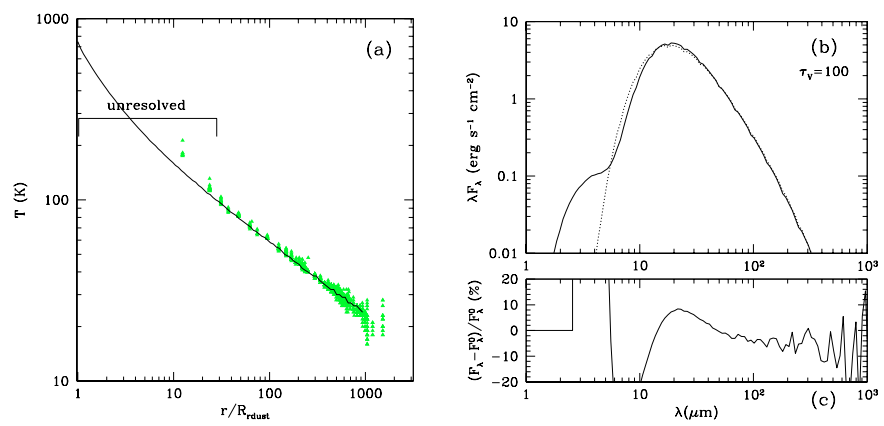

Fig. A.2. Ivezić test with $\tau_{\mathrm{V}}=100$, using $10^{7} \mathrm{~L}$-packets. The envelope is represented by $20000 \mathrm{SPH}$ particles and a global grid of $N_{\text {CELLS }}=1310$ RT cells (with $\bar{N}=15$ ), but no additional star grid around the star. a) The triangles represent the temperatures of the individual RT cells plotted against radius, whilst the solid line represents the benchmark calculation. The global grid on its own fails to capture the temperature profile close to the star. b) The solid line shows the SED computed here, and the dotted line shows the benchmark calculation. c) The percentage difference between the flux computed here and the benchmark calculation.
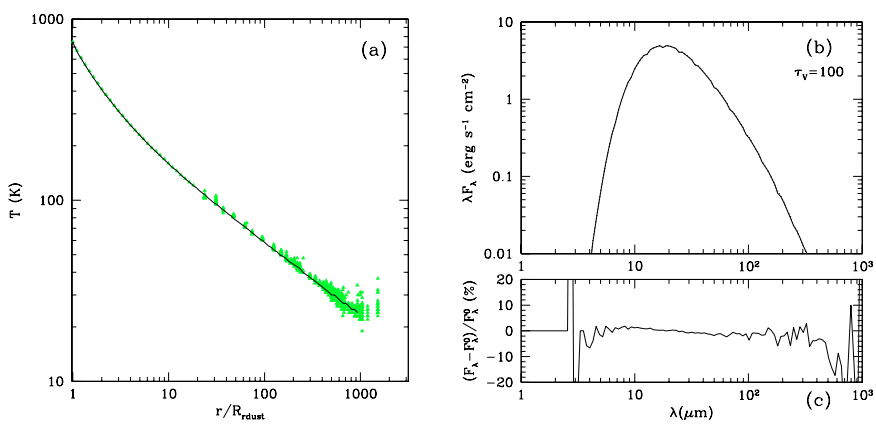

Fig. A.3. Same as Fig. A.2 but with an additional star grid around the star, i.e. a volume devoid of matter inside $R_{\mathrm{DEST}}$ and 30 concentric spherical RT cells between $R_{\mathrm{DEST}}$ and $R_{\mathrm{SG}}=20 R_{\mathrm{DEST}}$. Agreement with the benchmark calculation is now very good. Small differences are due to statistical noise. 